\title{
On the trace of the ring of integers of an abelian number field
}

\author{
by
}

\author{
KURT GIRSTMAIR (Innsbruck)
}

1. Introduction. Let $K, L$ be algebraic number fields with $K \subseteq L$, and $\mathcal{O}_{K}, \mathcal{O}_{L}$ their respective rings of integers. We consider the trace map

$$
T=T_{L / K}: L \rightarrow K
$$

and the $\mathcal{O}_{K}$-ideal $T\left(\mathcal{O}_{L}\right) \subseteq \mathcal{O}_{K}$. By $I(L / K)$ we denote the group index of $T\left(\mathcal{O}_{L}\right)$ in $\mathcal{O}_{K}$ (i.e., the norm of $T\left(\mathcal{O}_{L}\right)$ over $\mathbb{Q}$ ). It seems to be difficult to determine $I(L / K)$ in the general case. If $K$ and $L$ are absolutely abelian number fields, however, we obtain a fairly explicit description of the number $I(L / K)$. This is a consequence of our description of the Galois module structure of $T\left(\mathcal{O}_{L}\right)$ (Theorem 1). The case of equal conductors $f_{K}=f_{L}$ of the fields $K, L$ is of particular interest. Here we show that $I(L / K)$ is a certain power of 2 (Theorems 2, 3,4).

2. Basic notions. Let $d \in \mathbb{N}$ and $\xi_{d}=e^{2 \pi i / d}$. Then $\mathbb{Q}_{d}=\mathbb{Q}\left(\xi_{d}\right)$ is the $d$ th cyclotomic field. If $K$ is an absolutely abelian number field, we put $K_{d}=K \cap \mathbb{Q}_{d}$. By

$$
\xi_{d, K}=T_{\mathbb{Q}_{d} / K_{d}}\left(\xi_{d}\right)
$$

we denote the trace of the root of unity $\xi_{d}$ over $K_{d}$. Let $G_{K}=\operatorname{Gal}(K / \mathbb{Q})$ be the Galois group of $K$ over $\mathbb{Q}$ and $\mathbb{Z} G_{K}$ its integral group ring. For a number $m \in \mathbb{N}$ write

$$
m^{*}=\prod\{p ; p \mid m\},
$$

i.e., $m^{*}$ is the maximal square-free divisor of $m$. Let, in particular, $m=f_{K}$ be the conductor of $K$. Then $\mathcal{O}_{K}$ has a uniquely determined decomposition into indecomposable $\mathbb{Z} G_{K}$-modules, viz.

$$
\mathcal{O}_{K}=\bigoplus_{m^{*}|d| m} \mathbb{Z} G_{K} \xi_{d, K}
$$

(see [3], [4]). 
For simplicity we write $\mathcal{O}_{m}=\mathcal{O}_{\mathbb{Q}_{m}}$ and $G_{m}=G_{\mathbb{Q}_{m}}$. If $k$ is an integer prime to $m$, we define $\sigma_{k} \in G_{m}$ by

$$
\sigma_{k}\left(\xi_{m}\right)=\xi_{m}^{k}
$$

Then $G_{m}=\left\{\sigma_{k} ; 1 \leq k \leq m,(k, m)=1\right\}$.

Suppose now that both fields $K, L, K \subseteq L$, are abelian. Let $X_{K}, X_{L}$ be the character groups of $G_{K}, G_{L}$, resp. The restriction map

$$
()_{K}: G_{L} \rightarrow G_{K}: \sigma \mapsto \sigma_{K}=\left.\sigma\right|_{K}
$$

is surjective, and it defines an injection

$$
X_{K} \rightarrow X_{L}: \chi \mapsto \chi \circ()_{K} .
$$

Hence we consider $X_{K}$ as a subgroup of $X_{L}$. For a character $\chi \in X_{K}$ let $f_{\chi}$ be the conductor of $\chi$. Then $f_{\chi}$ divides $m=f_{K}$. Moreover, if $d \in \mathbb{N}$, we write

$$
[d]=\{c \in \mathbb{N} ; c \mid d, d / c \text { square-free, }(c, d / c)=1\} .
$$

There is a decomposition of $X_{K}$ that corresponds to (1) in a canonical way (see [1]). Indeed,

$$
X_{K}=\bigcup_{m^{*}|d| m}^{\bullet}\left\{\chi \in X_{K} ; f_{\chi} \in[d]\right\},
$$

and

$$
\operatorname{rank}_{\mathbb{Z}}\left(\mathbb{Z} G_{K} \xi_{d, K}\right)=\left|\left\{\chi \in X_{K} ; f_{\chi} \in[d]\right\}\right|
$$

for each $d, m^{*}|d| m$.

3. Description of $T_{L / K}\left(\mathcal{O}_{L}\right)$ and $I(L / K)$. Let the above notations hold, in particular, let $K \subseteq L$ be abelian number fields with conductors $f_{K}=m, f_{L}=n$. If $d$ is a divisor of $m$, write

$$
\widehat{d}=d \prod\{p ; p \text { prime, } p \mid n, p \nmid m\} .
$$

THEOREM 1. In the above situation the following assertions hold:

$$
T_{L / K}\left(\mathcal{O}_{L}\right)=\bigoplus_{m^{*}|d| m} \mathbb{Z} G_{K} h_{d} \xi_{d, K},
$$

with $h_{d}=[L: K] /\left[L_{\hat{d}}: K_{d}\right] ; h_{d}$ is an integer whenever $\xi_{d, K} \neq 0$.

$$
I(L / K)=\prod_{m^{*}|d| m} h_{d}^{r_{d}},
$$

with $r_{d}=\operatorname{rank}_{\mathbb{Z}}\left(\mathbb{Z} G_{K} \xi_{d, K}\right)=\left|\left\{\chi \in X_{K} ; f_{\chi} \in[d]\right\}\right|$.

Corollary. Let $m \mid n$. For $K=\mathbb{Q}_{m}, L=\mathbb{Q}_{n}$,

$$
T\left(\mathcal{O}_{n}\right)=n / \widehat{m} \cdot \mathcal{O}_{m}
$$




$$
I\left(\mathbb{Q}_{n} / \mathbb{Q}_{m}\right)=(n / \widehat{m})^{\varphi(m)},
$$

$\varphi$ denoting Euler's function.

We turn to the special case of equal conductors, so $K \subseteq L$ and $f_{K}=$ $f_{L}=n$. Write

$$
H=\operatorname{Gal}(L / K), \quad H_{d}=\operatorname{Gal}\left(L / L_{d}\right), \quad d \mid n .
$$

Suppose, moreover, that $q$ is a prime number and $[L: K]$ a power of $q$. Put $e=\max \left\{k ; 2^{k} \mid n\right\}$ (i.e., the 2-exponent of $n$ ). If $e \geq 1$, define $j, l \in$ $\{1, \ldots, n\}$ by the congruences

$$
\begin{aligned}
& j \equiv-1 \bmod 2^{e}, \quad l \equiv-1+2^{e-1} \bmod 2^{e}, \\
& j \equiv l \equiv 1 \bmod n / 2^{e} .
\end{aligned}
$$

THEOREM 2. In this situation the following assertions are equivalent:

(i) $I(L / K)>1$;

(ii) $q=2, e \geq 3$, and either $H \cap H_{n^{*}}=\left\langle\sigma_{j, L}\right\rangle \neq$ \{id $\}$ or $H \cap H_{n^{*}}=$ $\left\langle\sigma_{l, L}\right\rangle \neq\{\mathrm{id}\}$.

Remark. Let $\left(K, L_{n^{*}}\right)$ be the composite of the subfields $K, L_{n^{*}}$ of $L$. Then assertion (ii) can be restated as

(iii) $q=2, e \geq 3,\left[L:\left(K, L_{n^{*}}\right)\right]=2$, and either $\operatorname{Gal}\left(L /\left(K, L_{n^{*}}\right)\right)=\left\langle\sigma_{j, L}\right\rangle$ or $\operatorname{Gal}\left(L /\left(K, L_{n^{*}}\right)\right)=\left\langle\sigma_{l, L}\right\rangle$.

This is clear by Galois theory.

Theorem 3. Let $K \subseteq L, f_{K}=f_{L}=n, e \geq 3$, and let $[L: K]$ be a power of 2. Suppose that the equivalent conditions (i), (ii) of Theorem 2 are satisfied. If $H \cap H_{n^{*}}=\left\langle\sigma_{j, L}\right\rangle$ put $k=j$, otherwise put $k=l$. Then the numbers $h_{d}$ of Theorem 1 take the following values:

$$
h_{d}= \begin{cases}2 & \text { if } \sigma_{k, L_{d}}=\mathrm{id}, \\ 1 & \text { else. }\end{cases}
$$

In particular, $h_{d}=2$ for all $d$ with $n^{*}|d| n / 2^{e-1}$, and

$$
2^{\left[K_{n / 2} e: \mathbb{Q}\right]}|I(L / K)| 2^{[K: \mathbb{Q}]} .
$$

Corollary. In the situation of Theorem 3 let $L=\mathbb{Q}_{n}$. Then

$$
T_{\mathbb{Q}_{n} / K}\left(\mathcal{O}_{n}\right)=2 \cdot \mathcal{O}_{K_{n / 2} e} \oplus \bigoplus\left\{\mathbb{Z} G_{K} \xi_{d, K} ; n^{*}|d| n, 4 \mid d\right\}
$$

and $I(L / K)=2^{\left[K_{n / 2^{e}}: \mathbb{Q}\right]}$.

Theorems 2 and 3 also yield a description of $T\left(\mathcal{O}_{L}\right)$ and $I(L / K)$ for arbitrary abelian number fields $K \subseteq L$ of equal conductor $n$. As above, let $H=\operatorname{Gal}(L / K)$ and $H_{(p)}$ be the $p$-Sylow group of $H$ (p prime). Let $L^{(2)}$ be the fixed field of $\prod\left\{H_{(p)} ; p \neq 2\right\}$ (thus $\operatorname{Gal}\left(L^{(2)} / K\right)$ is isomorphic to $\left.H_{(2)}\right)$. 
THEOREM 4. In the above situation,

$$
T_{L / K}\left(\mathcal{O}_{L}\right)=T_{L^{(2)} / K}\left(\mathcal{O}_{L^{(2)}}\right) .
$$

Hence the structure of $T_{L / K}\left(\mathcal{O}_{L}\right)$ and the value of $I(L / K)$ are given by Theorems 2 and 3 applied to $K \subseteq L^{(2)}$.

\section{Proofs}

Proof of Theorem 1. First we show

$$
T\left(\mathcal{O}_{L}\right)=\bigoplus_{n^{*}|c| n} \mathbb{Z} G_{K} h_{c} \xi_{c, K}
$$

with $h_{c}=[L: K] /\left[L_{c}: K_{c}\right]$. Indeed, if $n^{*}|c| n$, then

$$
T_{L / K_{c}}\left(\xi_{c, L}\right)=T_{K / K_{c}}\left(T_{L / K}\left(\xi_{c, L}\right)\right)=\left[K: K_{c}\right] T_{L / K}\left(\xi_{c, L}\right),
$$

and

This yields

$$
T_{L / K_{c}}\left(\xi_{c, L}\right)=T_{L_{c} / K_{c}}\left(T_{L / L_{c}}\left(\xi_{c, L}\right)\right)=\left[L: L_{c}\right] \xi_{c, K}
$$

$$
T_{L / K}\left(\xi_{c, L}\right)=\left(\left[L: L_{c}\right] /\left[K: K_{c}\right]\right) \xi_{c, K}=h_{c} \xi_{c, K} .
$$

Hence $T\left(\mathbb{Z} G_{L} \xi_{c, L}\right)=\mathbb{Z} G_{L} T\left(\xi_{c, L}\right)=\mathbb{Z} G_{L} h_{c} \xi_{c, K}=\mathbb{Z} G_{K} h_{c} \xi_{c, K}$. We obtain

$$
T\left(\mathcal{O}_{L}\right)=\sum_{n^{*}|c| n} \mathbb{Z} G_{K} h_{c} \xi_{c, K}
$$

This sum, however, is direct, due to $\mathbb{Z} G_{K} h_{c} \xi_{c, K} \subseteq \mathbb{Z} G_{L} \xi_{c, L}$ and formula (1). Therefore (3) holds. For the time being, fix $c$ with $n^{*}|c| n$, and put $d=(c, m)$. Then $K_{d}=K_{c}$ and

$$
\xi_{c, K}=T_{\mathbb{Q}_{d} / K_{d}}\left(T_{\mathbb{Q}_{c} / \mathbb{Q}_{d}}\left(\xi_{c}\right)\right) .
$$

Moreover, formula (34) in [1] yields

$$
T_{\mathbb{Q}_{c} / \mathbb{Q}_{d}}\left(\xi_{c}\right)= \begin{cases} \pm \sigma_{k}\left(\xi_{d}\right) & \text { if } d \in[c], \\ 0 & \text { otherwise }\end{cases}
$$

$k$ being a certain number prime to $d$. From (4), (5) we conclude that $\xi_{c, K} \neq$ 0 only if $d \in[c]$, i.e., $c=\widehat{d}$. In this case $h_{c}=h_{d}$, and (4), (5) imply $\mathbb{Z} G_{K} \xi_{c, K}=\mathbb{Z} G_{K} \xi_{d, K}$. We obtain from (3)

$$
T\left(\mathcal{O}_{L}\right)=\bigoplus_{m^{*}|d| m} \mathbb{Z} G_{K} h_{d} \xi_{d, K}
$$

Observe that $\mathbb{Z} G_{K} h_{d} \xi_{d, K} \subseteq \mathcal{O}_{K}, m^{*}|d| m$. Hence (1) implies $h_{d} \mathbb{Z} G_{K} \xi_{d, K} \subseteq$ $\mathbb{Z} G_{K} \xi_{d, K}$. If $\xi_{d, K} \neq 0, \mathbb{Z} G_{K} \xi_{d, K}$ is a free $\mathbb{Z}$-module of $\mathbb{Z}$-rank $\geq 1$, and $h_{d}$ must be an integer. This concludes the proof of (i). Assertion (ii) follows from (i), (1), and (2). 
Proof of the Corollary (of Theorem 1). For each $d$ with $m^{*}|d| m$ the number $h_{d}$ equals $\varphi(n) \varphi(d) /(\varphi(m) \varphi(\widehat{d}))=\varphi(n) / \varphi(\widehat{m})=n / \widehat{m}$. Since $h_{d}$ does not depend on the choice of $d$, the assertions follow from (1).

Proof of Theorem 2. Let $n^{*}|d| n$. By Galois theory, $\operatorname{Gal}\left(L / K_{d}\right)=$ $\operatorname{Gal}\left(L / K \cap L_{d}\right)=\left\langle H, H_{d}\right\rangle=H H_{d}$. Moreover, $\left|H H_{d}\right|=|H|\left|H_{d}\right| /\left|H \cap H_{d}\right|$. After a short calculation this yields

$$
h_{d}=\left|H \cap H_{d}\right| .
$$

Suppose that (ii) holds. Then $h_{n^{*}}=2$, by (6). Formula (1) shows that

$$
\mathcal{O}_{K_{n^{*}}}=\mathbb{Z} G_{K} \xi_{n^{*}, K}
$$

which yields $r_{n^{*}}=\operatorname{rank}_{\mathbb{Z}} \mathcal{O}_{K_{n^{*}}} \geq 1$. From Theorem 1(ii), we infer that $I(L / K)>1$.

Conversely, assume (i). We shall show in the subsequent steps (a)-(d) that (ii) holds.

(a) There is a number $d, n^{*}|d| n$, such that $H \cap H_{d} \neq\{$ id $\}$. Because of $H_{d} \subseteq H_{n^{*}}, H \cap H_{n^{*}} \neq\{\mathrm{id}\}$, too. Since $|H|$ is a power of $q, H \cap H_{n^{*}}$ is a non-trivial subgroup of the $q$-Sylow group $H_{n^{*}, q}$ of $H_{n^{*}}$.

(b) Suppose that $q \neq 2$ or $q=2, e \leq 2$. We show that $H_{n^{*}, q}$ is a cyclic group. Put

$$
J=\operatorname{Gal}\left(\mathbb{Q}_{n} / L\right), \quad J_{n^{*}}=\operatorname{Gal}\left(\mathbb{Q}_{n} / \mathbb{Q}_{n^{*}}\right) .
$$

Then $J J_{n^{*}}=\operatorname{Gal}\left(\mathbb{Q}_{n} / L_{n^{*}}\right)$. The restriction map

$$
()_{L}: \operatorname{Gal}\left(\mathbb{Q}_{n} / L_{n^{*}}\right) \rightarrow \operatorname{Gal}\left(L / L_{n^{*}}\right)=H_{n^{*}}: \sigma \mapsto \sigma_{L}
$$

is surjective; because of $(J)_{L}=1$ we get $H_{n^{*}}=\left(J J_{n^{*}}\right)_{L}=\left(J_{n^{*}}\right)_{L}$. We assert that the $q$-Sylow group $J_{n^{*}, q}$ of $J_{n^{*}}$ is cyclic. Indeed, the Chinese Remainder Theorem yields a canonical isomorphism

$$
\psi: G_{n} \rightarrow \prod_{p \mid n}\left(\mathbb{Z} / p^{e_{p}} \mathbb{Z}\right)^{\times},
$$

$e_{p}=\max \left\{k ; p^{k} \mid n\right\}$ being the $p$-exponent of $n$. But $\psi$ maps $J_{n^{*}}$ onto $\prod_{p \mid n}\{\bar{k} ; k \equiv 1 \bmod p\}$, whose $q$-Sylow group is

$$
\{\bar{k} ; k \equiv 1 \bmod q\} \times \prod_{p \neq q}\{\overline{1}\} .
$$

Since $q \geq 3$ or $q=2, e \leq 2$, this group is cyclic.

(c) Again suppose $q \neq 2$ or $q=2, e \leq 2$. If $e_{q}=1,\left|J_{n^{*}}\right|=n / n^{*} \not \equiv$ $0 \bmod q$; thus $\left|H_{n^{*}}\right| \not \equiv 0 \bmod q$ and $\left|H \cap H_{n^{*}}\right| \not \equiv 0 \bmod q$, contrary to step (a). Hence assume $e_{q} \geq 2$. Then $H_{n / q} \subseteq H_{n^{*}}$. Furthermore, $\left|J_{n / q}\right|=q$, which gives $\left|H_{n / q}\right| \mid q$ and $H_{n / q} \subseteq H_{n^{*}, q}$. However, $H_{n^{*}, q}$ is cyclic by step (b), and $H \cap H_{n^{*}}$ is a non-trivial subgroup, by (a). This requires $H_{n / q} \subseteq H \cap H_{n^{*}}$ $\subseteq H$. Therefore $K \subseteq L_{n / q}$, which is impossible, due to $f_{K}=n$. 
(d) Step (c) has shown that $q=2$ and $e \geq 3$. Let $\sigma_{k, L} \in H \cap H_{n^{*}}$, $\sigma_{k, L} \neq \mathrm{id}$. Since there is an epimorphism ()$_{L}: J_{n^{*}, 2} \rightarrow H_{n^{*}, 2}$, we can assume that $\sigma_{k} \in J_{n^{*}, 2}$, i.e., $k \equiv 1 \bmod n / 2^{e}$. It is well-known that $k$ satisfies one of the congruences

$$
k \equiv \pm 5^{b} \bmod 2^{e}, \quad 1 \leq b \leq 2^{e-2}
$$

(see, e.g., [2], p. 43). Suppose that $b<2^{e-2}$. Then there is a divisor $c$ of $2^{e-3}$ such that

$$
5^{b c} \equiv 1+2^{e-1} \bmod 2^{e}
$$

(loc. cit.). We get $k^{c} \equiv( \pm 1)^{c}\left(1+2^{e-1}\right) \bmod 2^{e}$. If $c>1$, this yields $\sigma_{k}^{c} \in J_{n / 2} \backslash\{\mathrm{id}\}$. But $\left|J_{n / 2}\right|=2$, thus $J_{n / 2}=\left\langle\sigma_{k}^{c}\right\rangle$ and $H_{n / 2}=\left\langle\sigma_{k, L}^{c}\right\rangle \subseteq H$, contrary to $f_{K}=n$. Therefore $c=1$, and $k \equiv \pm\left(1+2^{e-1}\right) \bmod 2^{e}$. The case $k \equiv 1+2^{e-1} \bmod 2^{e}$ is impossible again. Altogether, we have shown that $b=2^{e-2}, k \equiv-1 \bmod 2^{e}$, or that $k \equiv-1-2^{e-1} \equiv-1+2^{e-1} \bmod 2^{e}$. This implies $H \cap H_{n^{*}}=\left\langle\sigma_{j, L}\right\rangle \neq\{\mathrm{id}\}$ or $H \cap H_{n^{*}}=\left\langle\sigma_{l, L}\right\rangle \neq\{\mathrm{id}\}$.

Proof of Theorem 3 and the Corollary. Let $k$ be as assumed and $H \cap H_{n^{*}}=\left\langle\sigma_{k, L}\right\rangle \neq \mathrm{id}$. Consider a number $d$ with $n^{*}|d| n$. Then $H \cap H_{d} \subseteq H \cap H_{n^{*}}$; by (6) we get $h_{d} \neq 1$ if and only if $\sigma_{k, L} \in H_{d}$, which means $\sigma_{k, L_{d}}=\mathrm{id}$. Obviously this is the case if $4 \nmid d$. We have shown

$$
\begin{aligned}
2 \cdot \mathcal{O}_{k} & \subseteq T\left(\mathcal{O}_{L}\right) \\
& \subseteq \bigoplus\left\{\mathbb{Z} G_{K} 2 \xi_{d, K} ; n^{*}|d| n / 2^{e-1}\right\} \oplus \bigoplus\left\{\mathbb{Z} G_{K} \xi_{d, K} ; 2 n^{*}|d| n\right\} \\
& =2 \cdot \mathcal{O}_{K_{n / 2} e} \oplus \bigoplus\left\{\mathbb{Z} G_{K} \xi_{d, K} ; 2 n^{*}|d| n\right\} .
\end{aligned}
$$

This gives

$$
2^{\left[K_{n / 2}: \mathbb{Q}\right]}|I(L / K)| 2^{[K: \mathbb{Q}]} .
$$

In the case $L=\mathbb{Q}_{n}$, the last inclusion can be replaced by equality.

Proof of Theorem 4. We have $\left[L: L^{(2)}\right]=|H| /\left|H_{(2)}\right|$, which is an odd number. For this reason there exists a chain of intermediate fields

$$
L^{(2)} \subseteq \ldots \subseteq L^{\prime} \subseteq L^{\prime \prime} \subseteq \ldots \subseteq L
$$

such that $\left[L^{\prime \prime}: L^{\prime}\right]$ is an odd prime power. All of these fields have conductor $n$. So Theorem 2 implies $T_{L^{\prime \prime} / L^{\prime}}\left(\mathcal{O}_{L^{\prime \prime}}\right)=\mathcal{O}_{L^{\prime}}$, whence $T_{L / L^{(2)}}\left(\mathcal{O}_{L}\right)=$ $\mathcal{O}_{L^{(2)}}$. Finally,

$$
T_{L / K}\left(\mathcal{O}_{L}\right)=T_{L^{(2)} / K}\left(T_{L / L^{(2)}}\left(\mathcal{O}_{L}\right)\right)=T_{L^{(2)} / K}\left(\mathcal{O}_{L^{(2)}}\right)
$$

\section{References}

[1] K. Girstmair, Dirichlet convolution of cotangent numbers and relative class number formulas, Monatsh. Math. 110 (1990), 231-256. 
[2] K. Ireland and M. Rosen, A Classical Introduction to Modern Number Theory, Springer, New York 1982.

[3] H. W. Leopoldt, Über die Hauptordnung der ganzen Elemente eines abelschen Zahlkörpers, J. Reine Angew. Math. 201 (1959), 119-149.

[4] G. Lettl, The ring of integers of an abelian number field, ibid. 404 (1990), 162-170.

INSTITUT FÜR MATHEMATIK

UNIVERSITÄT INNSBRUCK

TECHNIKERSTR. $25 / 7$

A-6020 INNSBRUCK, ÖSTERREICH

Received on 10.2.1992

and in revised form on 25.3 .1992 